\title{
Identifying Human Face Profiles with Semi-Local Integral Invariants
}

\author{
Jun Sato and Roberto Cipolla \\ Department of Engineering, University of Cambridge, Cambridge CB2 1PZ, England
}

\begin{abstract}
In this paper, we propose a method for identifying human face profiles by using invariant representations of image curves. In particular, we show that semi-local invariants are very useful for matching profile curves and identifying human faces. A method for finding face profiles from the changes in apparent contours of faces is also considered. The results of some experiments with real images of human faces show the power of the proposed method.
\end{abstract}

\section{Introduction}

Identifying human faces is very important for many applications in computer vision, such as security systems. From ancient times, artists often used human profiles to depict human faces. In fact human face profiles provide important cues to identify an individual person as shown in Fig. 1.

Although human faces are curved surfaces, we can assume that the face profiles are the projections of planar curves, if the viewing axis is perpendicular to the plane of symmetry, and if the distance to the face from the viewer is large enough. Under this assumption, the distortions of the profile caused by viewer motions are described by similarity transformations. Thus, if the similarity invariants are available from the profile curves, we can use these invariants for identifying individual faces.

The question is what sort of invariants are available from the profile curves. Unfortunately, existing invariants $[1,6,9-11]$ in computer vision suffer from problems due to the occlusion of objects, the existence of image noise and the requirement of point or line correspondences. That is, if the invariants are defined locally they are sensitive to noise, and if the invariants are defined globally they suffer from the occlusion and the correspondence problems. The existence and importance of semi-local invariants has recently been shown $[7,8]$. In this paper, we show that semi-local invariants under the similarity group can be applied successfully for identifying individual human face profiles.

We first consider invariant representations of human face profiles. We next investigate how to find the canonical profile of a face under rotational motions of the face. The preliminary results of face identification experiments are shown. The distinguishability of the proposed method is also investigated. 


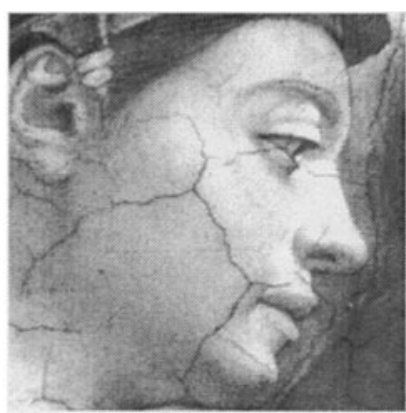

(a)

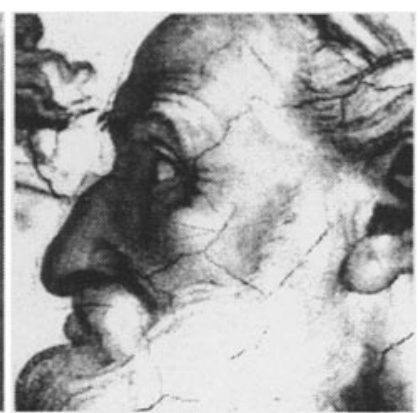

(b)

Fig. 1. Face profiles. (a) and (b) are example pictures of human face profiles painted by Michelangelo in the Sistina Chapel in Rome. Profiles have often been used to depict human faces.

\section{Invariant Representation of Face Profiles}

Consider a face to have $3 \mathrm{D}$ bilateral symmetry. The direction of the face and the longitudinal axis define a plane of symmetry (see Fig. 2). The ray from the camera center touches the curved surface of the face. The contour generator is defined on the curved surface as a set of tangent points of the ray to the surface, and separates the visible and occluded parts on the surface. The projection of the contour generator onto the image plane is an apparent contour [4]. In general, the contour generator slips over the surface as the relative position and orientation between the camera and the surface changes.

If the viewing axis is perpendicular to the plane of symmetry as shown in Fig. 2, we call the apparent contour a canonical profile of the face. If the distance from the viewer to the face is large compared to the depth of the face, then the contour generator on the face can be considered a planar curve (or approximately a planar curve) which lies on the plane of symmetry. Since the plane of symmetry is perpendicular to the viewing axis, the distortions of the profile curve of a face caused by the changes in viewpoint are described by planar similarity transformations. Thus, if we can extract invariants under the similarity group, then we could use these invariants for identifying individual human face profiles. The problem is what sort of similarity invariants can be applied for this task. To recognise faces reliably the invariants should not be sensitive to noise, should not suffer under partial occlusions and should have enough distinguishability. However, in general, local invariants such as differential invariants [11] are sensitive to noise and are poor in distinguishability, while global invariants such as moment invariants $[5,9]$ suffer from the occlusion problem. To cope with these problems we next introduce semi-local integral invariants, which are less sensitive to noise than classical differential invariants, and unlike moment invariants do not suffer from the occlusion problem.

The semi-local integral invariants were introduced by Sato and Cipolla [7] and Bruckstein et al. [2], and are used for matching image curves and extracting symmetry axes of planar and $3 \mathrm{D}$ bilateral symmetry [8]. The idea of semi-local 


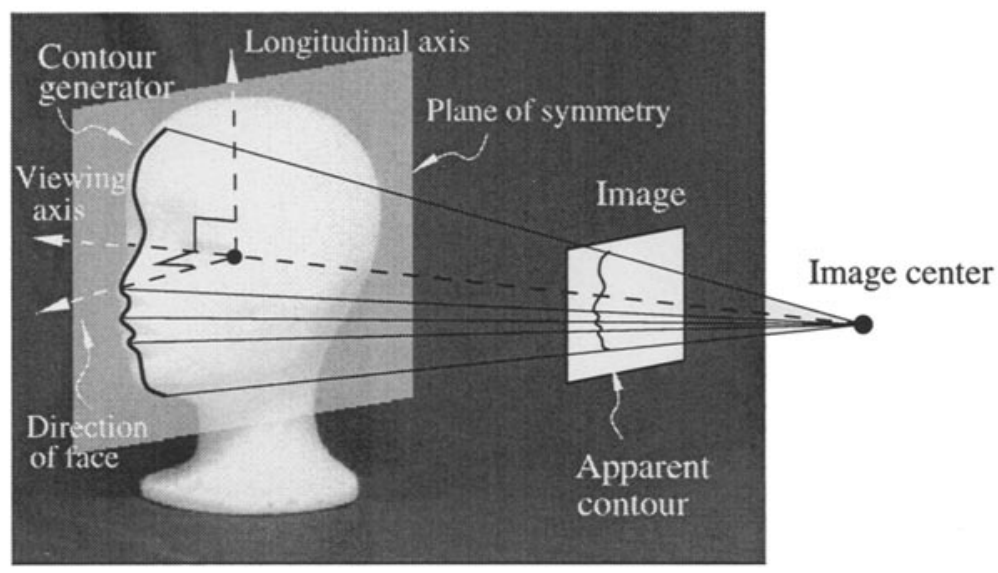

Fig. 2. Apparent contours under the weak perspective assumption. If the viewing axis is perpendicular to the direction of face and the longitudinal axis under weak perspective, the distortion of the apparent contour can be described by similarity transformations.

integral invariants is to define integral invariants based on invariant parameterisations of the group.

Since as we have seen similarity invariants are required for identifying human face profiles, we next consider the semi-local integral invariants under the similarity group. It is known that the invariant parameterisation, $s$, under similarity transformations can be described by the Euclidean curvature, $\kappa$, and the Euclidean arc-length, $v$, as follows:

$$
d s=\kappa d v
$$

where $d s$ and $d v$ are the differentials of $s$ and $v$ respectively. By integrating an invariant function, $F$, with respect to $s$ along the curve with interval of $[-\Delta s, \Delta s]$, we have a semi-local invariant under similarity transformations:

$$
I\left(s_{1}\right)=\int_{s_{1}-\Delta s}^{s_{1}+\Delta s} F d s
$$

If we choose the function $F$ carefully, the integral formula (2) can be solved analytically, and the resulting invariants have simple forms. For example, if we substitute $F(s)=\left\langle C\left(s_{1}+\Delta s\right)-C\left(s_{1}-\Delta s\right), C_{s}(s)\right\rangle$ into (2), we have the following invariant:

$$
I_{1}\left(s_{1}\right)=\left\langle\boldsymbol{C}\left(s_{1}+\Delta s\right)-\boldsymbol{C}\left(s_{1}-\Delta s\right), \boldsymbol{C}\left(s_{1}+\Delta s\right)-\boldsymbol{C}\left(s_{1}-\Delta s\right)\right\rangle
$$

where $C_{s}$ denotes the derivative of $C$ with respect to $s$, and $\langle$,$\rangle denotes the$ scalar product of two vectors. Since $I_{1}$ is a relative invariant of weight 1 , an absolute invariant can be computed by taking a ratio of two semi-local invariants as follows:

$$
I_{2}\left(s_{1}\right)=\frac{\left\langle C\left(s_{1}+\Delta s_{1}\right)-C\left(s_{1}-\Delta s_{1}\right), C\left(s_{1}+\Delta s_{1}\right)-C\left(s_{1}-\Delta s_{1}\right)\right\rangle}{\left\langle C\left(s_{1}+\Delta s_{2}\right)-C\left(s_{1}-\Delta s_{2}\right), C\left(s_{1}+\Delta s_{2}\right)-C\left(s_{1}-\Delta s_{2}\right)\right\rangle}
$$




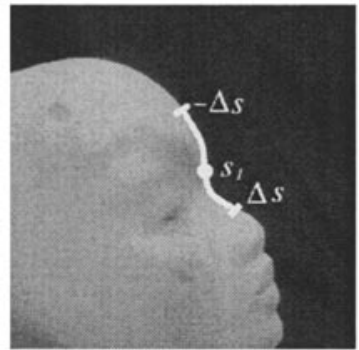

(a)

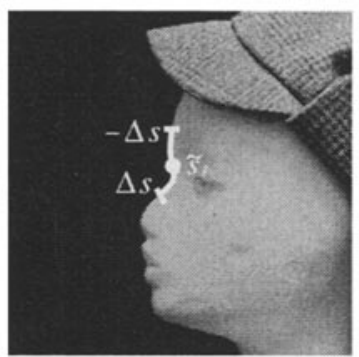

(b)

Fig. 3. Semi-local invariant. The interval of integration can be identified uniquely from invariant arc-length, $s$. That is, if $s_{1}$ and $\widetilde{s}_{1}$ correspond to each other, the interval $[-\Delta s, \Delta s]$ with respect to $s$ corresponds to the same interval $[-\Delta s, \Delta s]$ with respect to $\tilde{s}$ in the second image. Even though the curve is partially occluded (second image), the semi-local integral invariants can be defined on the remaining parts of the curve.

We now summarise the properties of semi-local integral invariants:

1. Occlusion:

Even if a part of the curve is occluded, the semi-local invariants can be defined on the remaining parts (visible parts) of the curve. Thus, these invariants do not suffer from the occlusion problem.

\section{Noise Sensitivity:}

Since the semi-local invariants enable us to reduce the order of derivatives from that of group curvatures to that of group arc-length, they require lower order derivatives than differential invariants. The semi-local integral invariants are therefore less sensitive to noise than classical differential invariants.

\section{Distinguishability:}

Since the new invariants are computed from a certain interval of a curve (not from a point), their distinguishability is much higher than local invariants which are computed at a single point on the curve.

\section{Finding Profiles from Apparent Contours}

Up to now, we have shown that if we have face profiles, we can identify them by using semi-local integral invariants under similarity transformations. In general, however, we do not know whether the extracted apparent contour in an image is of the canonical profile or not. In this section, we describe how to find canonical profiles from the projected apparent contours of faces.

We assume that we do not have any rotation around the direction of face. The distortions of an apparent contour are therefore due to the rotations around the viewing axis and the longitudinal axis. As it is shown in [4], the change in position, $\mathbf{r}_{t}$, of a point on a contour generator is described by the curvature of the surface in the direction of the viewing ray, $\kappa$, the normal to the surface, $\mathbf{n}$, and the change in ray direction, $\mathbf{p}_{t}$ as follows:

$$
\left|\mathbf{r}_{t}\right|=\frac{\left\langle\mathbf{p}_{t}, \mathbf{n}\right\rangle}{\kappa}
$$




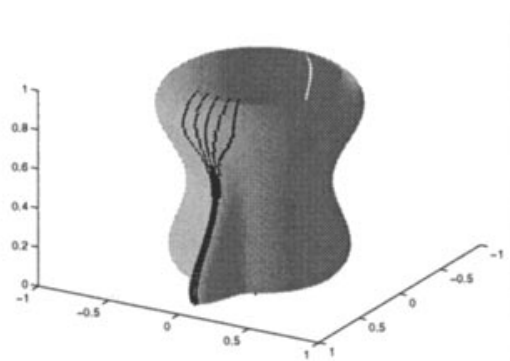

(a)

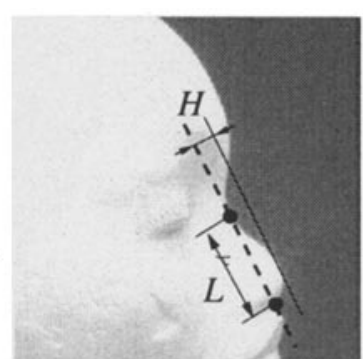

(b)

Fig. 4. Apparent contours and Face profiles. (a) shows an an artificial face model and the changes in contour generator (black lines) caused by the rotation of the face around the longitudinal axis. The contour generator on the nose is almost fixed on the surface. (b) shows an image of a model face. The dashed line is a bitangent line to the contour curve of the face. $L$ and $H$ are the length and the height of the nose in the image.

From (5) we have the following observations:

1. Under rotations around the viewing axis, $\left\langle\mathbf{p}_{t}, \mathbf{n}\right\rangle=0$ and $\left|\mathbf{r}_{t}\right|=0$, so the contour generator does not slip on the surface of a face.

2. Since $\kappa$ of a nose is large, the changes in contour generator of a nose part are negligible under rotational motions around the longitudinal axis as shown in Fig. 4 (a).

Thus, the contour generator of a nose part can be approximately considered as a fixed planar curve in the space. This property of contour generator is useful for finding the canonical profiles of face. Let us consider a bitangent line to the bottoms of the nose of a face in the image as shown in Fig. 4 (b). We define the length, $L$, of a nose as a distance between two bitangent points, and the height, $H$ of the nose as a distance between the bitangent line and the top of the nose. If we rotate the face around the viewing axis and the longitudinal axis, the ratio, $R=\frac{H}{L}$, between the height, $H$, and the length, $L$, changes. Since the contour generator of a nose part is considered as a planar curve, the apparent contour is of the canonical profile, if the ratio, $R$, takes a maximum.

\section{Experiments}

\subsection{Procedure for Identifying Face Profiles}

We now summarise the procedure for identifying human face profiles.

1. B-spline curves are fitted [3] to the apparent contour of a face.

2. The profile curve of the face is found by rotating the face maximising $R$.

3. The similarity arc-length and the similarity semi-local integral invariant (4) with an arbitrary but constant $\Delta s$ are computed at all points on the curves, and subsequently plotted on an invariant graph with similarity arc-length as horizontal axis and the semi-local integral invariant as vertical axis. The derived curve on the graph is an invariant signature up to a horizontal shift. 
4. To match curves we simply shift one invariant signature horizontally minimising the total difference between the two signatures.

5. Face profiles are identical if the two signatures match each other.

\subsection{Preliminary Results}

We next show the results of some experiments. Fig. 5 (a) and (b) show images of a model face observed from two different viewpoints. The extracted contour curves are shown by the white lines in each image. In Fig. 5 (b), we have not only distortion but also occlusions of image curves. The semi-local invariants are computed at every point on the curves, and plotted on the graph as shown in Fig. 5 (c). In this and the following experiments, we chose $\Delta s_{1}=0.15$ and $\Delta s_{2}=1.2$ for the intervals of integration. In this graph, the horizontal and the vertical axes are the arc-length and the semi-local invariants under similarity transformations. The solid and dashed lines are the invariant signatures from Fig. 5 (a) and (b) respectively. As we can see in this graph, the semi-local invariants are quite useful for identifying human face profiles even under occlusions. Fig. 6 (a) and (b) show the profile images of a real human face, and (c) shows the extracted invariant signatures. Again the computed signatures are very stable and useful for identifying the human face. In Fig. 6 (d), (e) and (f), we show the results from another example. Even if the face expressions are different in (d) and (e), the computed invariant signatures are identical as shown in (f), and are thus useful. This is because, the changes in shape of foreheads and noses are small under the changes in expression.

\subsection{Distinguishability}

We next show the distinguishability of the proposed signatures. Fig. 7 (a) and (b) show the two different profiles, and (c) shows their invariant signatures. As we can see, the signatures are very different, and it is quite easy to distinguish these two faces, while if we use classical differential invariants, they are difficult to distinguish because of the high sensitivity to image noise.

\section{Conclusions}

In this paper, we have proposed an efficient method for identifying human face profiles from an invariant representation of image curves. Semi-local invariants under similarity transformations are exploited for matching image curves and identifying profiles. Since the semi-local invariants are less sensitive to noise and have higher distinguishability than traditional differential invariants, the proposed method is useful for identifying human faces reliably. The method is implemented and tested on real face images. Even if the face expressions are different the extracted invariant signatures are stable, and useful for identifying faces. 


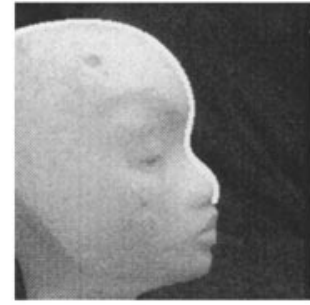

(a)

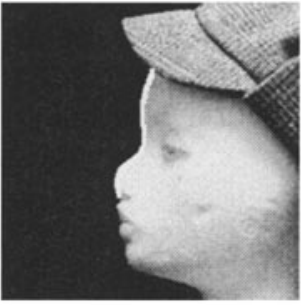

(b)

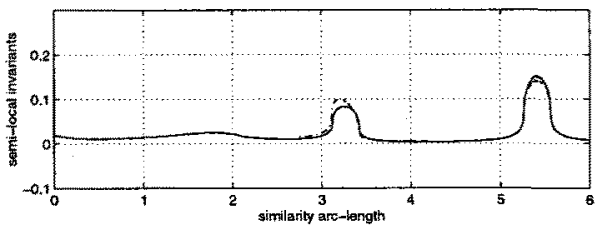

(c)

Fig. 5. Results from a model face. (a) and (b) show images of a model face observed from two different viewpoints. The extracted contour curves are shown by the white lines in each image. In Fig. 5 (b), we have not only distortion but also occlusions of image curves. The solid and dashed lines in (c) show invariant signatures computed from the curves in (a) and (b) by using the semi-local invariants. The horizontal and the vertical axes are the similarity arc-length and the semi-local invariants under similarity transformations.

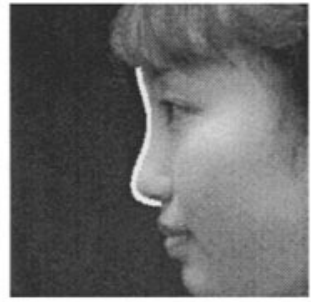

(a)

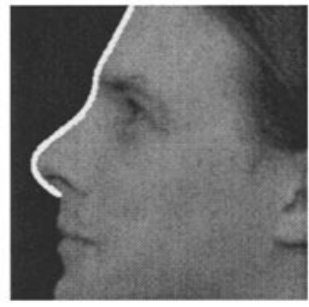

(d)

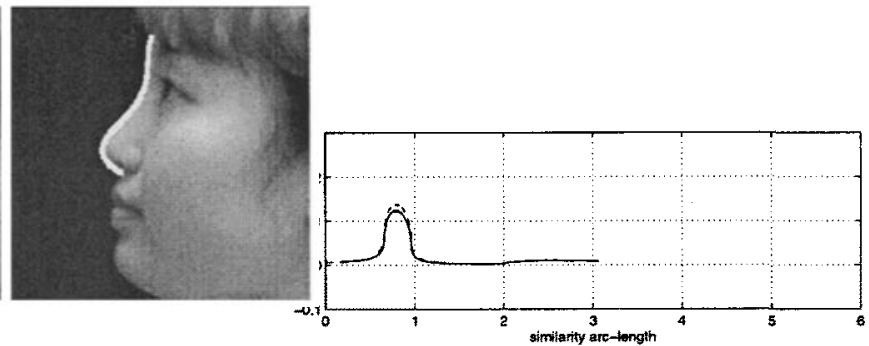

(b)

(c)

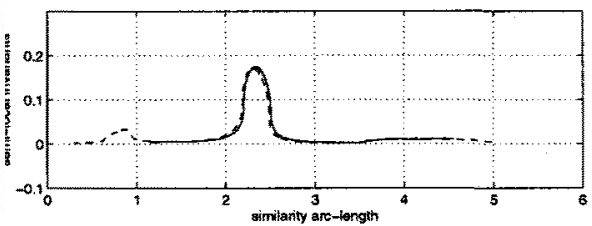

(f)

Fig. 6. Results from a real face. (a) and (b) show images of a human face observed from two different viewpoints. The extracted contour curves are shown by the white lines in each image. The solid and dashed lines in (c) show invariant signatures computed from the curves in (a) and (b) by using the semi-local invariants. The horizontal and the vertical axes are the similarity arc-length and the semi-local invariants under similarity transformations. (d), (e) and (f) show the result from another example. Note the face expressions in (d) and (e) are different. 


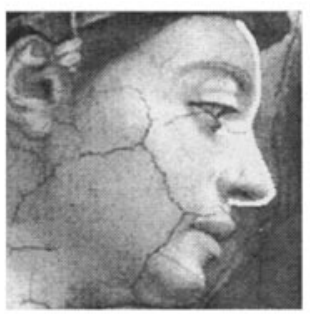

(a)

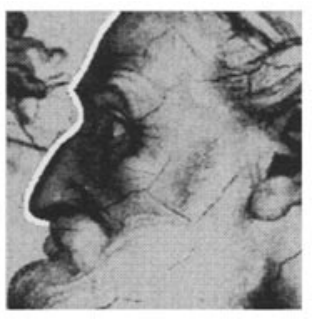

(b)

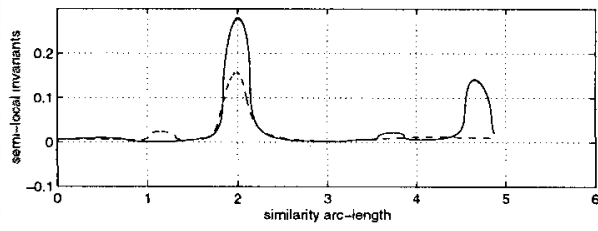

(c)

Fig. 7. Distinguishability of semi-local invariants. (a) and (b) show images of two different profiles. The white lines show the extracted contour curves of the profiles. The solid and the dashed lines in (c) show invariant signatures of these contour curves.

The signatures are very different from each other.

\section{References}

1. E.B. Barrett and P.M. Payton. General methods for determining projective invariants in imagery. Computer Vision, Graphics and Image Processing, 53(1):46-65, 1991.

2. A.M. Bruckstein, R.J. Holt, A.N. Netravali, and T.J. Richardson. Invariant signatures for planar shape recognition under partial occlusion. Computer Vision, Graphics and Image Processing, 58(1):49-65, 1993.

3. T.J. Cham and R. Cipolla. Automated B-spline curve representation with MDLbased active contours. In Proc. British Machine Vision Conference, volume 2, pages 363-372, Edinburgh, September 1996.

4. R. Cipolla and A. Blake. Surface shape from the deformation of apparent contours. International Journal of Computer Vision, 9(2):83-112, 1992.

5. M. Hu. Visual pattern recognition by moment invariants. IRE Transaction on Information Theory, IT-8:179-187, February 1962.

6. J.L. Mundy and A. Zisserman. Geometric Invariance in Computer Vision. MIT Press, Cambridge, USA, 1992.

7. J. Sato and R. Cipolla. Affine integral invariants and matching of curves. In Proc. International Conference on Pattern Recognition, volume 1, pages 915-919, Vienna, Austria, August 1996.

8. J. Sato and R. Cipolla. Affine integral invariants for extracting symmetry axes. In Proc. British Machine Vision Conference, volume 1, pages 63-72, Edinburgh, September 1996.

9. G. Taubin and D.B. Cooper. Object recognition based on moment (or algebraic) invariants. In J.L. Mundy and A. Zisserman, editors, Geometric Invariance in Computer Vision, pages 375-397. MIT Press, 1992.

10. L.J. Van Gool, T. Moons, E. Pauwels, and A. Oosterlinck. Semi-differential invariants. In J.L. Mundy and A. Zisserman, editors, Geometric Invariance in Computer Vision, pages 157-192. MIT Press, 1992.

11. I. Weiss. Projective invariants of shapes. In Proc. Image Understanding workshop, volume 2, pages 1125-1134, 1988. 